\title{
Identification and Characterization of Long Non-Coding RNAs in Osteogenic Differentiation of Human Adipose-Derived Stem Cells
}

\author{
Guangxin Huang Yan Kang Zhiyu Huang Zhiqi Zhang Fangang Meng \\ Weishen Chen Ming Fu Weiming Liao Ziji Zhang
}

Department of Joint Surgery, First Affiliated Hospital of Sun Yat-sen University, Guangzhou, China

\section{Key Words}

Long noncoding RNAs • Osteogenesis • Adipose-derived stem cells • Microarray

\begin{abstract}
Background/Aims: Long noncoding RNAs (IncRNAs) play important roles in stem cell differentiation. However, their role in osteogenesis of human adipose-derived stem cells (ASCs), a promising cell source for bone regeneration, remains unknown. Here, we investigated the expression profile and potential roles of IncRNAs in osteogenic differentiation of human ASCs. Methods: Human ASCs were induced to differentiate into osteoblasts in vitro, and the expression profiles of IncRNAs and mRNAs in undifferentiated and osteogenic differentiated ASCs were obtained by microarray. Bioinformatics analyses including subgroup analysis, gene ontology analysis, pathway analysis and co-expression network analysis were performed. The function of IncRNA H19 was determined by in vitro knockdown and overexpression. Quantitative reverse transcription polymerase chain reaction was utilized to examine the expression of selected genes. Results: We identified 1,460 upregulated and 1,112 downregulated IncRNAs in osteogenic differentiated human ASCs as compared with those of undifferentiated cells (Fold change $\geq 2.0, P<0.05$ ). Among these, 94 antisense IncRNAs, 85 enhancer-like IncRNAs and 160 lincRNAs were further recognized. We used 12 IncRNAs and 157 mRNAs to comprise a coding-non-coding gene expression network. Additionally, silencing of H19 caused a significantly increase in expression of osteogenesis-related genes, including ALPL and RUNX2, while a decrease was observed after $\mathrm{H} 19$ overexpression. Conclusion: This study revealed for the first time the global expression profile of IncRNAs involved in osteogenic differentiation of human ASCs and provided a foundation for future investigations of IncRNA regulation of human ASC osteogenesis.




\section{Introduction}

Osteoporosis is a common, age-related skeletal disease characterized by the loss of bone mass and deficits in bone quality, leading to low bone strength and increased risk of fractures [1]. Current treatments for osteoporosis are predominantly focused on pharmacological drugs, although transplantation of mesenchymal stem cells (MSCs) has become an attractive approach during the past decade [2-4]. Bone marrow mesenchymal stem cells (BMSCs) have been considered an ideal cell source for bone regeneration for years; however, increasing evidence suggests that the proliferative ability and osteogenic differentiation capability of BMSCs decrease with age or under other osteoporotic conditions. Meanwhile, this is always accompanied by the generation of additional marrow adipose tissue [5-7]. Recently, adiposederived stem cells (ASCs) have been studied extensively as an alternative cell source to BMSCs due to their widespread sources, easy access and multipotency [3, 8]. More importantly, ASCs isolated from aged or osteoporotic humans and animals maintain their osteogenic differentiation capability, making them more promising for autologous cell-based therapy for osteoporosis [9-11]. Their osteogenic differentiation is regulated at transcriptional, posttranscriptional and epigenetic levels. However, the precise molecular mechanisms related to osteogenesis require further investigation.

Long noncoding RNAs (lncRNAs) are a novel subset of noncoding RNAs that are over 200 nucleotides in length. Increasing evidence shows that IncRNAs are involved in diverse physiological processes, as well as pathological processes, via different mechanisms [12, 13]. Interestingly, numerous lncRNAs are restricted to specific cell lineages, indicating their potential roles in the determination of cell fate [14]. Previous studies suggested that lncRNAs play important roles in MSC differentiation, including several publications that investigated the expression and potential role of lncRNAs in the osteogenesis of human BMSCs, mouse C3H10T1/2 MSCs and other types of MSCs [15-22]. Most recently, one study demonstrated an inhibitory role of IncRNA-MIR31HG in osteogenic differentiation of human ASCs under an inflammatory microenvironment [23]. However, there remains a lack of data concerning the global expression and potential function of IncRNAs in the differentiation of human ASCs into osteoblasts.

In this study, we compared the expression profiles of lncRNAs between osteogenic differentiated human ASCs and undifferentiated cells by microarray analysis, followed by bioinformatics analyses and in vitro experiments used to explore the potential roles of these differentially expressed lncRNAs in osteogenesis. These findings provided a primary foundation for future investigations of lncRNA regulation of ASC osteogenesis.

\section{Materials and Methods}

\section{Cell culture}

The use of human cells was approved by the Ethics Committee of The First Affiliated Hospital of Sun Yat-sen University, China ([2013]C-260). Human ASCs derived from the fat tissue of different healthy donors (aged 18-45-years, $n=4$ ) were purchased from Cyagen (Suzhou, China). ASCs were maintained in OriCell ${ }^{\mathrm{TM}}$ Human MSC Growth Medium containing 10\% MSCs-Qualified Fetal Bovine Serum (FBS), 1\% PenicillinStreptomycin and $1 \%$ Glutamine (Cyagen, China) under $37^{\circ} \mathrm{C}, 5 \% \mathrm{CO}_{2}$ conditions. The medium was replaced every 3 days, and when cells reached $80 \%$ confluence, they were passaged using $0.25 \%$ trypsin/EDTA (Gibco, USA).

Osteogenic differentiation of ASCs

Third passage human ASCs were seeded into six-well plates at a density of $2 \times 10^{4} \mathrm{cells} / \mathrm{cm}^{2}$, and cells were cultured in standard growth medium until they reached $50 \%$ to $70 \%$ confluence. The medium was then changed to OriCell ${ }^{\mathrm{TM}}$ human ASC Osteogenic Differentiation Medium (Cyagen) containing 10\% FBS, 1\% Penicillin-Streptomycin, $10 \mathrm{mM}$ L-glutamine, $50 \mu \mathrm{M}$ L-ascorbic acid, $10 \mathrm{mM}$ beta-glycerophosphate and $100 \mathrm{nM}$ dexamethasone. The differentiation medium was carefully replaced every 3 days. After a 14- 


\section{Cellular Physiology Cell Physiol Biochem 2017;42:1037-1050

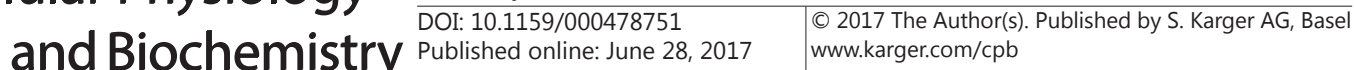 \\ Published online: June 28, 2017 is}

day induction, cells were harvested for RNA analysis and meanwhile stained with alizarin red (Cyagen) to measure mineralization by microscopy according to manufacturer's instructions. Undifferentiated human ASCs were used as controls. For detection of dynamic expression of lncRNA H19, human ASC cultures were induced to osteogenic differentiation for 21 days, and RNA was isolated from the cells at days $0,7,14$, and 21 for quantitative real-time reverse transcription PCR (qRT-PCR).

\section{Transient transfection}

The $H 19$ siRNA and scramble negative control siRNA were purchased from RiboBio (Guangzhou, China). The target sequences of the three siRNAs against $H 19$ are as follows: si-H19-1, GACACCATCGGAACAGCAG; si-H19-2, CCCGTCCCTTCTGAATTTA; si-H19-3, CGTGACAAGCAGGACATGA. The H19 overexpression plasmid pEX-3-H19 was constructed by cloning the entire H19 coding fragment into the Xhol/EcoRI site of the pEX-3 vector. The pEX-3-H19 plasmid and pEX-3 empty plasmid were designed and synthesized by GenePharma (Shanghai, China). Human ASCs cultured in antibiotic-free growth medium were allowed to grow to $60 \%$ to $70 \%$ confluence prior to transfection with $50 \mathrm{nM}$ siRNA or $50 \mathrm{nM}$ plasmid using Lipofectamine ${ }^{\circledR} 2000$ Transfection Reagent (Invitrogen, USA) according to manufacturer's instructions. After 48 hours, cells were induced to osteogenic differentiation for 3 days as above and total RNA was extracted for qRT-PCR analysis.

\section{RNA isolation and quality control}

Total RNA from human ASCs was extracted using Trizol reagent (Invitrogen) according to the instructions of manufacturer. A NanoDrop ND-1000 spectrophotometer was used for measuring RNA quantity and quality, and agarose gel electrophoresis was used to examine RNA integrity.

\section{Microarray hybridization}

Human LncRNA Microarray V3.0 (Arraystar, USA) used in this study is a commercial array (Agilent Design ID: Agilent-045997) designed for the global profiling of human lncRNAs and mRNAs. Approximately 30,586 IncRNAs and 26,109 mRNAs can be detected using this microarray. According to the most highlyrespected public transcriptome databases, including Refseq, UCSC knowngenes, Genecode and landmark publications, IncRNAs were carefully constructed on the microarray. Each individual transcript was identified by a specific exon or splice junction probe. Negative probes and positive probes were also included in the array for hybridization quality control.

Sample preparation and array hybridization were modified from the protocol for Agilent One-Color Microarray-Based Gene Expression Analysis (Agilent Technology, USA) and utilized the Arraystar Flash RNA Labeling Kit (Arraystar, USA). Briefly, purified RNA was amplified and transcribed into fluorescent cRNA along the entire length of the transcripts by utilizing a mixture of oligo dT and random primers to avoid 3' bias. After measuring concentration and specific activity, the fluorescent cRNAs were hybridized onto the IncRNA array slide, followed by the arrays being washed, fixed and scanned using a DNA microarray scanner (Agilent Technology). Feature Extraction Software v11.0.1.1 (Agilent Technology) was used to extract raw data from the acquired array images.

\section{qRT-PCR}

For detection of mRNAs and lncRNAs, $1 \mu \mathrm{g}$ of total RNA was reversely transcribed to cDNA using random hexamer primer and SuperScript III Reverse Transcriptase (Invitrogen). Then, qRT-PCR was performed on a Bio-Rad CFX96 System (Bio-Rad, USA) using SYBR Premix Ex Taq ${ }^{\mathrm{TM}}$ II (Takara, Japan) according to manufacturer's instructions. The reaction volume contained $10 \mu \mathrm{L}$ SYBR Premix Ex Taq II (2x), $0.8 \mu \mathrm{L}$ of forward primer and reverse primer $(10 \mu \mathrm{M}), 2 \mu \mathrm{L}$ template cDNA and $6.4 \mu \mathrm{L} \mathrm{dH}_{2} \mathrm{O}$ to a final volume of $20 \mu \mathrm{L}$. The reaction conditions were as follows: denaturation at $95^{\circ} \mathrm{C}$ for 5 seconds, followed by 40 cycles of $95^{\circ} \mathrm{C}$ for 5 seconds and $60^{\circ} \mathrm{C}$ for 30 seconds. Melting curve analysis was performed from $65^{\circ} \mathrm{C}$ to $95^{\circ} \mathrm{C}$ with an increment of $0.5^{\circ} \mathrm{C}$. All qRT-PCR reactions were performed in triplicate. GAPDH expression was used to normalize expression of IncRNAs and mRNAs. The $2^{-\Delta \Delta C t}$ method was used to calculate relative gene expression. Primers for qRT-PCR were designed with Primer 5.0 and BLAST was used to determine individual specificity. Amplification efficiency was determined by standard curve for each primer. The templates for standard curve were the PCR products which were synthetized from cDNA of undifferentiated human ASCs and purified via agarose gel electrophoresis. Ten 10-fold serial dilutions of templates were prepared and 1/ (dilution factor) was used as a concentration. Standard curve was obtained by plotting Ct value on the X-axis 


\section{Cellular Physiology Cell Physiol Biochem 2017;42:1037-1050 and Biochemistry Published $\begin{aligned} & \text { DOI: 10.1159/000478751 } \\ & \text { (c) } 2017 \text { The Author(s). Published by S. Karger AG, Basel } \\ & \text { www.karger.com/cpb }\end{aligned}$ \\ Huang et al.: LncRNAs in Osteogenesis of Human ASCs}

and $\log$ (template concentration) on the Y-axis. The amplification efficiency was calculated as $10^{-1 / \text { slope }}-1$ (for all online suppl. material, see www.karger.com/doi/10.1159/ 000478751, Table S1).

\section{Bioinformatics analysis and statistical analysis}

After quantile normalization of the raw data, IncRNAs and mRNAs flagged as "Present" or "Marginal" in at least four out of eight samples were used for subsequent data analysis with GeneSpring GX v12.1 software package (Agilent Technology). Differentially expressed IncRNAs and mRNAs between two samples in each pair of cells were identified through Fold Change filtering (Fold Change $\geq 2.0$ ). The significance of differences in expression levels between the two groups was evaluated by paired $t$-test. And $P$-value filtering $(P<0.05)$ was used to identify significant differentially expressed transcripts. Hierarchical Clustering and combined analysis were performed using homemade scripts. Euclidean distance and average linkage rule were used for clustering. To explore the potential functions of the dysregulated lncRNAs, classification and subgroup analysis was performed. Both gene ontology (GO) analysis and pathway analysis were carried out to determine the roles of the differentially expressed mRNAs. Fisher's exact test was used to test the significance of GO terms and Pathway identifiers enrichment in the differentially expressed gene list. Pearson's product-moment correlation coefficient was used to assess correlations between the expression of lncRNAs and co-expressed coding transcripts. Those dysregulated lncRNAs and mRNAs exhibiting Pearson's coefficients $>0.99$ were selected to construct a coding-non-coding gene co-expression network (CNC network) using Cytoscape. Microarray and bioinformatics analyses were performed by KangChen Bio-tech (Shanghai, China). For qRT-PCR validation experiment, Spearman's correlation coefficients were used to correlate the fold changes measured by qPCR and microarray. In cases of multiple-group analysis, one-way analysis of variance (ANOVA) followed by Dunnett- $t$ test was conducted. A value of $P<0.05$ was considered statistically significant.

\section{Results}

Differentially expressed IncRNAs in osteogenic differentiated human ASCS

After a 14-day osteogenic induction, alizarin red staining indicated that calcium deposition was positive, and the expression of osteogenesis-related genes, including collagen type I alpha 1 chain (COL1A1), alkaline phosphatase liver/bone/kidney (ALPL) and runtrelated transcription factor $2(R U N X 2)$ was significantly increased in differentiated cells as compared with undifferentiated human ASCs (Fig. 1). These results confirmed osteogenic differentiation.

We identified a total of 17,246 lncRNAs expressed in human ASCs according to microarray analysis. Hierarchical clustering analysis showed homogeneous lncRNA expression profiles within the same group and distinct expression profiles between the two groups (Fig. 2A). Comparing the osteogenic differentiated ASCs to undifferentiated cells, 1,460 lncRNAs were upregulated and 1,112 lncRNAs were downregulated (Fold change $\geq 2.0, P<0.05$ ) (Fig. 2B).

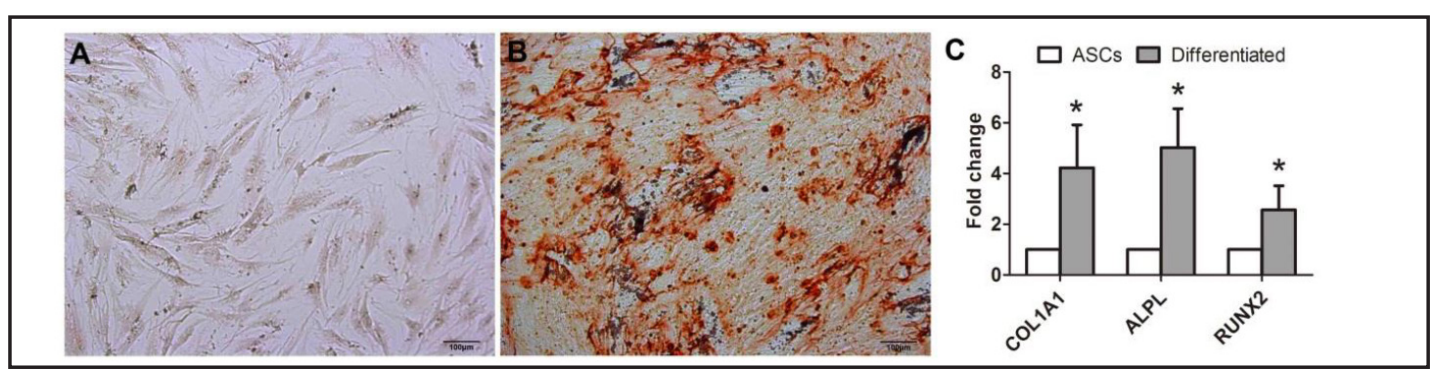

Fig. 1. Analysis of osteogenic differentiation of human ASCs. Alizarin red staining was performed for undifferentiated human ASCs (A) and differentiated cells after a 14-day osteogenic induction (B) (100x). (C) Osteogenesis-related genes expression was determined by qRT-PCR. Gene expression is presented as relative fold change by normalized to GAPDH expression and to undifferentiated cells control values using the $2^{-\triangle \Delta C \mathrm{Ct}}$ method. Values represent the mean \pm SD of four different pairs of samples. ${ }^{*} P<0.05,{ }^{* *} P<0.01$ vs. undifferentiated ASCs as determined by paired $t$-test. 
Fig. 2. LncRNA and mRNA expression profiles based on microarray data. Hierarchical Clustering results show a distinguishable IncRNA (A) and mRNA (C) expression profile between the two groups and homogeneity within the same group. "Value" stands for the gene expression. The number " 5 " represents a relatively low expression level and is represented by the color green, whereas the number " 9 " represents a relatively high expression level and is represented by the color red in cluster maps. "Count" represents the number of genes that exhibited the same expression level. A total of 17,246 lncRNAs and 21,591 mRNAs were detected in human ASCs. Volcano Plots of IncRNAs (B) and mRNAs (D) in differentiated cells versus undifferentiated ASCs. Red squares in the plots represent the differentially expressed transcripts with statistical significance (Fold change $\geq$ 2.0, $P<0.05$ ). Compared to undifferentiated ASCs, 2,572 IncRNAs and 2,586 mRNAs were significantly dysregulated.

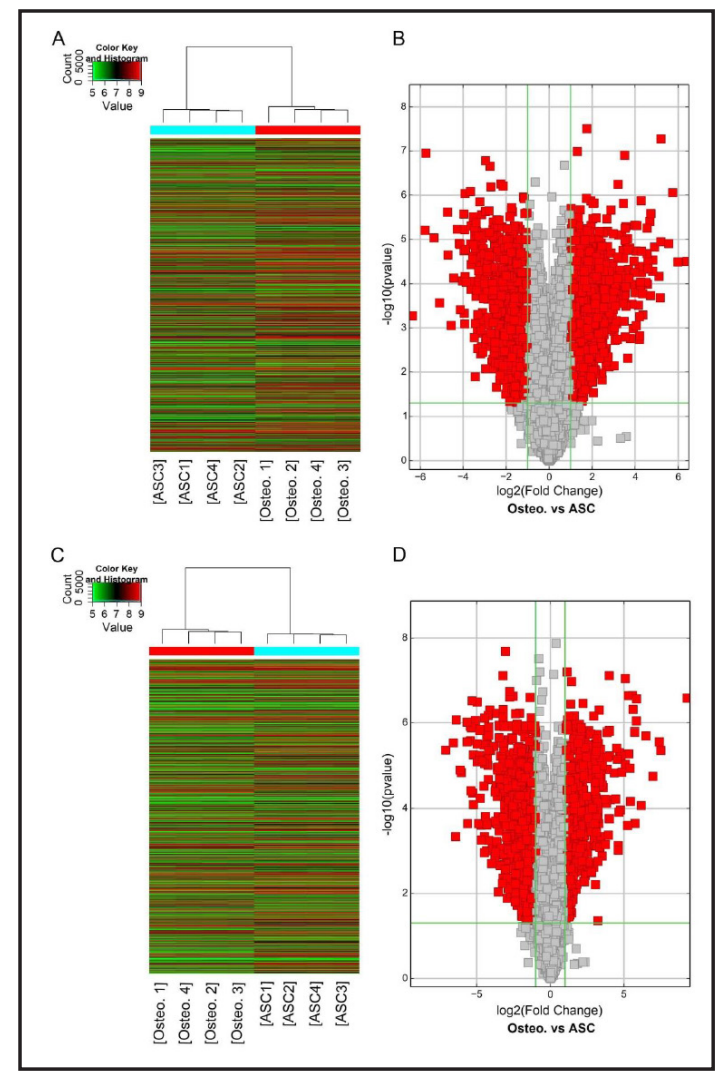

Fig. 3. Classification analysis of dysregulated lncRNAs. (A) LncRNAs were classified into six categories including intergenic, natural antisense, intronic antisense, intron sense-overlapping, exon sense-overlapping and bidirectional according to the relative position between IncRNAs and their nearby genes. The graph showed the
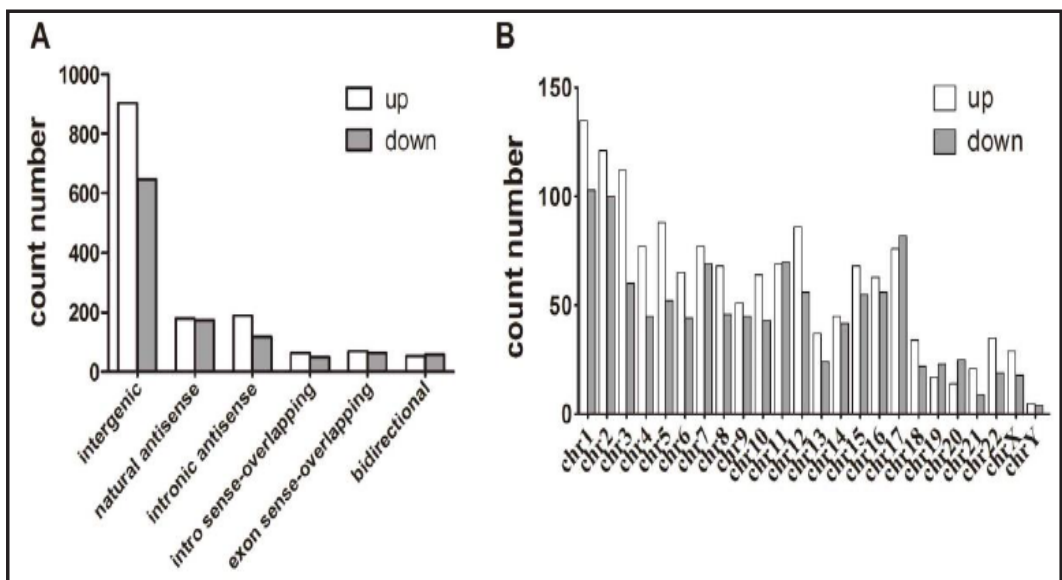
numbers of differentially expressed lncRNAs in each category. (B) Chromosome distribution analysis shows the numbers of both upregulated and downregulated lncRNAs in different chromosomes.

Among these, the expression of 88 lncRNAs were altered $>10$-fold in each pair of samples, including 55 upregulated and 33 downregulated genes. Specifically, the most upregulated lncRNA was uc002lbc.1 (Fold change: 84.37) and the most downregulated was uc.247+ (Fold change: 82.20) (Table 1). The microarray data was submitted to the Gene Expression Omnibus (Accession number: GSE89330).

\section{Differentially expressed mRNAs in osteogenic differentiated human ASCs}

A total of 21,591 mRNAs were identified by microarray analysis, with the differentially expressed mRNA profile revealing that 946 mRNAs were upregulated and 1,640 mRNAs were downregulated during osteogenic differentiation (Fold change $\geq 2.0, P<0.05$ ) (Fig. 
2C-D). Of these, SLIT and NTRK like family member 1 (SLITRK1) was the most upregulated mRNA and Matrix metallopeptidase 3 (MMP3) was most downregulated (Table 2).

GO analysis showed that the upregulated mRNAs were highly enriched in extracellular matrix organization (ontology: biological process), extracellular space (ontology: cellular component) and peptidase regulator activity (ontology: molecular function), and that the downregulated mRNAs were highly enriched in system development (ontology: biological process), cell periphery (ontology: cellular component) and protein binding (ontology: molecular function) (see supplementary material, Fig. S1). Pathway analysis revealed that the most highly enriched pathway for upregulated mRNAs was the phosphoinositide 3-kinase (PI3K)/Akt signaling pathway and the most highly enriched pathway for downregulated mRNAs was the steroid biosynthesis network (see supplementary material, Fig. S2). Notably, there is mounting evidence suggesting that the PI3K/Akt pathway is involved in osteogenesis [24-26].

Classification and subgroup analysis of differentially expressed lncRNAs

To obtain general signatures of dysregulated IncRNAs, we analyzed upregulated and downregulated lncRNAs according to classification and chromosome distribution. Based on the relative position of lncRNAs and the nearby coding genes on chromosomes, IncRNAs were divided into six categories including intergenic, natural antisense, intronic antisense, intron sense-overlapping, exon sense-overlapping and bidirectional. In our microarray data, the majority $(60.34 \%)$ of the differentially expressed
Table 2. Top 15 differentially expressed mRNAs (differentiated vs. undifferentiated)

\begin{tabular}{|c|c|c|c|}
\hline Gene Symbol & Seqname & Fold change & $\mathrm{P}$-value \\
\hline SLITRK1 & NM_052910 & +628.32 & 0.00000026 \\
\hline CORIN & NM_006587 & +181.07 & 0.0000042 \\
\hline BZW2 & NM_014038 & +169.84 & 0.0000027 \\
\hline FAM153B & ENST00000253490 & +125.82 & 0.000017 \\
\hline C3orf58 & NM_173552 & +89.94 & 0.0000020 \\
\hline MMP7 & NM_002423 & +72.25 & 0.000084 \\
\hline STEAP4 & NM_024636 & +57.91 & 0.00000028 \\
\hline CIDEC & NM_022094 & +57.56 & 0.00000090 \\
\hline MRPS18B & NM_014046 & +54.39 & 0.00022 \\
\hline FRZB & NM_001463 & +53.18 & 0.0000016 \\
\hline ADH1A & NM_000667 & +48.80 & 0.00000048 \\
\hline 0R51A2 & ENST00000380371 & +48.12 & 0.00000023 \\
\hline GPR110 & NM_025048 & +43.57 & 0.000068 \\
\hline LPL & NM_000237 & +42.37 & 0.000011 \\
\hline SAA2 & NM_030754 & +39.70 & 0.0000052 \\
\hline MMP3 & NM_002422 & -139.40 & 0.0000042 \\
\hline PKIB & NM_032471 & -96.58 & 0.0000028 \\
\hline FAM181A & NM_138344 & -86.68 & 0.00046 \\
\hline KLK8 & ENST00000320838 & -84.958 & 0.00000084 \\
\hline PI16 & ENST00000373674 & -70.058 & 0.000012 \\
\hline F7 & NM_000131 & -66.79 & 0.000014 \\
\hline MMP10 & NM_002425 & -50.63 & 0.00000097 \\
\hline SPP2 & NM_006944 & -50.30 & 0.0000027 \\
\hline FCN2 & ENST00000291744 & -49.22 & 0.00022 \\
\hline GAGE2B & ENST00000381725 & -43.81 & 0.0000012 \\
\hline 0R7A10 & ENST00000248058 & -43.52 & 0.0000025 \\
\hline SLPI & NM_003064 & -42.37 & 0.000038 \\
\hline MFN2 & NM_001127660 & -41.49 & 0.0000013 \\
\hline SLC39A13 & NM_152264 & -40.05 & 0.00000030 \\
\hline LRSAM1 & NM_001005374 & -39.15 & 0.000042 \\
\hline
\end{tabular}


Fig. 4. Comparison of the expression of selected IncRNAs between microarray and qRT-PCR results. Ten lncRNAs (five upregulated and five downregulated) from microarray data were selected for validation by qRT-PCR in four paired undifferentiated and osteogenic differentiated human ASCs. For microarray analysis, the raw intensity of genes was quantile normalized. For qRT-PCR analysis, gene expression was normalized to GAPDH expression and calculated using the $2^{-\Delta \Delta C t}$ method. The heights of the columns in the

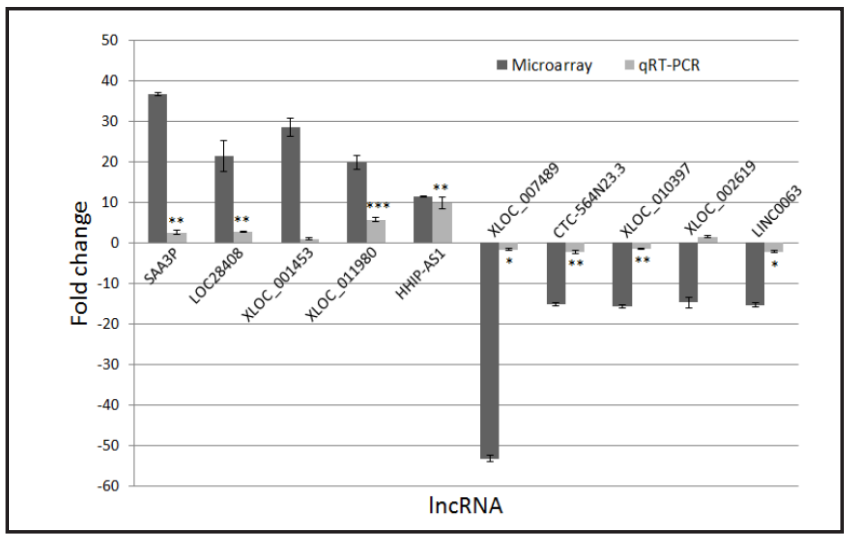
graph represent the mean fold changes (differentiated/undifferentiated) of expression. Paired $t$-test was used for comparison between undifferentiated and differentiated conditions. The expression of these lncRNAs as measured by microarray was significantly different between undifferentiated and differentiated cells $(P<0.001) .{ }^{*} P<0.05,{ }^{* *} P<0.01,{ }^{* * *} P<0.001$ indicated differentiated vs. undifferentiated samples in qRT-PCR results.

Table 3. Top 15 differentially expressed antisense lncRNAs and their nearby mRNAs

\begin{tabular}{lllll}
\hline Seqname & Fold change & Genome Relationship & Nearby Gene & Fold change \\
\hline ENST00000563280 & +26.52 & natural antisense & FOXC2 & +8.74 \\
NR_037595 & +11.36 & natural antisense & HHIP & +3.82 \\
AA321097 & -10.089 & intronic antisense & FOXP1 & -5.10 \\
ENST00000438380 & -10.01 & intronic antisense & NR6A1 & -3.74 \\
ENST00000444873 & -9.43 & intronic antisense & BCL11A & -23.11 \\
ENST00000503242 & -9.02 & intronic antisense & NREP & -3.18 \\
ENST00000439592 & +8.84 & intronic antisense & DMD & +3.19 \\
NR_026882 & -8.48 & intronic antisense & C11orf9 & -2.07 \\
ENST00000435932 & -8.39 & natural antisense & ELN & -2.28 \\
NR_037884 & +8.05 & natural antisense & ADH1A & +48.80 \\
ENST00000418335 & -7.96 & natural antisense & DPP4 & -3.26 \\
ENST00000534431 & -7.19 & natural antisense & KIAA1549L & -3.03 \\
ENST00000455081 & -7.02 & intronic antisense & MAPRE3 & -3.46 \\
ENST00000509939 & +6.98 & natural antisense & ADH1A & +48.80 \\
ENST00000444923 & +6.63 & intronic antisense & VWA5B1 & -2.74 \\
\hline
\end{tabular}

Table 4. Top 15 differentially expressed enhancer-like lncRNAs and their nearby mRNAs

\begin{tabular}{lllll}
\hline Seqname & Fold change & Genome Relationship & Nearby Gene & Fold change \\
\hline TCONS_00018338 & -41.20 & downstream & TCF7L2 & -2.05 \\
NR_034119 & +18.98 & downstream & EFNB2 & +8.19 \\
ENST00000447028 & -11.81 & upstream & SLC22A12 & -2.64 \\
ENST00000432961 & -10.84 & upstream & HES4 & +2.15 \\
ENST00000417705 & -9.98 & upstream & HES4 & +2.15 \\
ENST00000450938 & +9.21 & downstream & TLR4 & +2.88 \\
ENST00000457833 & -8.85 & downstream & THBS2 & -3.61 \\
ENST00000447481 & -8.51 & upstream & ENPP1 & +3.91 \\
ENST00000421509 & +7.05 & upstream & TLR4 & +2.88 \\
ENST00000436510 & -6.32 & downstream & IER5L & -2.56 \\
ENST00000443783 & -6.09 & upstream & ARHGAP8 & -2.35 \\
ENST00000427208 & -5.68 & upstream & SULT1C2 & -5.86 \\
ENST00000414039 & -5.63 & upstream & F3 & -2.42 \\
ENST00000449119 & +5.42 & upstream & FAM150B & -3.11 \\
ENST00000425666 & -5.40 & upstream & DIRAS2 & -2.05 \\
\hline
\end{tabular}

IncRNAs were intergenic IncRNAs (Fig. 3A). The upregulated and downregulated lncRNAs were dispersed in various chromosomes (Fig. 3B). 


\begin{tabular}{|c|c|c|}
\hline Cellular Physiology & Cell Physiol Biochem 2017; & 42:1037-1050 \\
\hline and Riochemictrv & 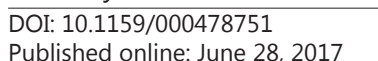 & $\begin{array}{l}\text { O } 2017 \text { The Author(s). Published by S. Karger AG, Basel } \\
\text { wwwk.kargerccom/cob }\end{array}$ \\
\hline
\end{tabular}

Table 5. Top 15 differentially expressed lincRNAs and their nearby mRNAs

\begin{tabular}{lllll}
\hline Seqname & Fold change & Genome Relationship & Nearby Gene & Fold change \\
\hline TCONS_00016098 & -53.13 & upstream & NR4A3 & +3.38 \\
TCONS_00018338 & -41.20 & downstream & TCF7L2 & -2.05 \\
TCONS_00003663 & +28.29 & upstream & SIX2 & +2.41 \\
TCONS_00024702 & +19.66 & upstream & BEAN1 & -31.45 \\
TCONS_00021794 & -15.67 & upstream & PCDH8 & -4.18 \\
TCONS_00005994 & -14.50 & upstream & CX3CR1 & +5.96 \\
TCONS_00025773 & -12.70 & upstream & TIMP2 & -2.07 \\
TCONS_00020439 & +11.61 & downstream & NCKAP1L & +2.35 \\
TCONS_00013698 & -8.66 & downstream & SCIN & -2.53 \\
TCONS_00008419 & -8.58 & downstream & ABLIM2 & -2.53 \\
TCONS_00008650 & +8.39 & downstream & SAP30 & +2.29 \\
TCONS_00018417 & +7.79 & upstream & AKR1E2 & -2.15 \\
TCONS_00011165 & +7.78 & upstream & BTN3A1 & -2.02 \\
TCONS_00011825 & -7.73 & upstream & OPN5 & -6.17 \\
TCONS_00001811 & +7.53 & upstream & BATF3 & +2.12 \\
\hline
\end{tabular}

Fig. 5. Construction of CNC network. Triangular nodes represent lncRNAs and circular nodes represent $\mathrm{mR}$ NAs. The sizes of nodes represent degrees of connectivity, with larger sizes indicating greater degrees. Solid lines represent positive correlations and dashed lines represent negative correlations between IncRNAs and mRNAs.

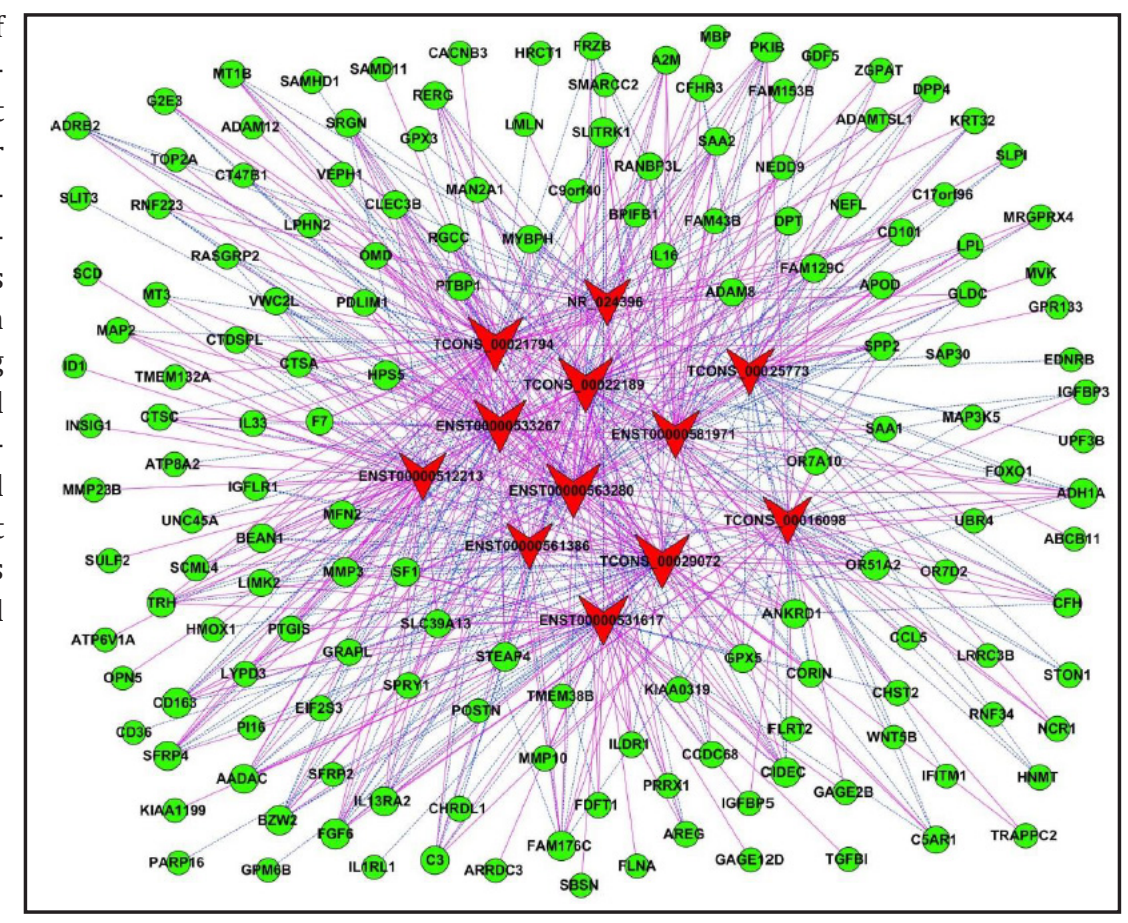

Differentially expressed IncRNAs were further classified into subgroups including antisense lncRNAs, enhancer-like lncRNAs and long intergenic non-coding RNAs (lincRNAs). The associated mRNAs were also identified to help predict the potential function of these dysregulated IncRNAs. Over 30\% of human transcripts have corresponding antisense IncRNAs and these IncRNAs exert biological functions by regulating their sense mRNAs [27]. Therefore, we identified 94 dysregulated antisense IncRNAs, as well as differentially expressed sense mRNAs, from our microarray data (Table 3). Additionally, according to a previous study [28], 85 enhancer-like lncRNAs were found to be differentially expressed during osteogenesis of human ASCs and the associated mRNAs were identified to explore the putative targets of these IncRNAs (Table 4). Moreover, based on Cabili's work [29], expression of 160 lincRNAs changed significantly at 14 days after osteogenic induction and the differentially expressed nearby coding genes were subsequently identified (Table 5). 

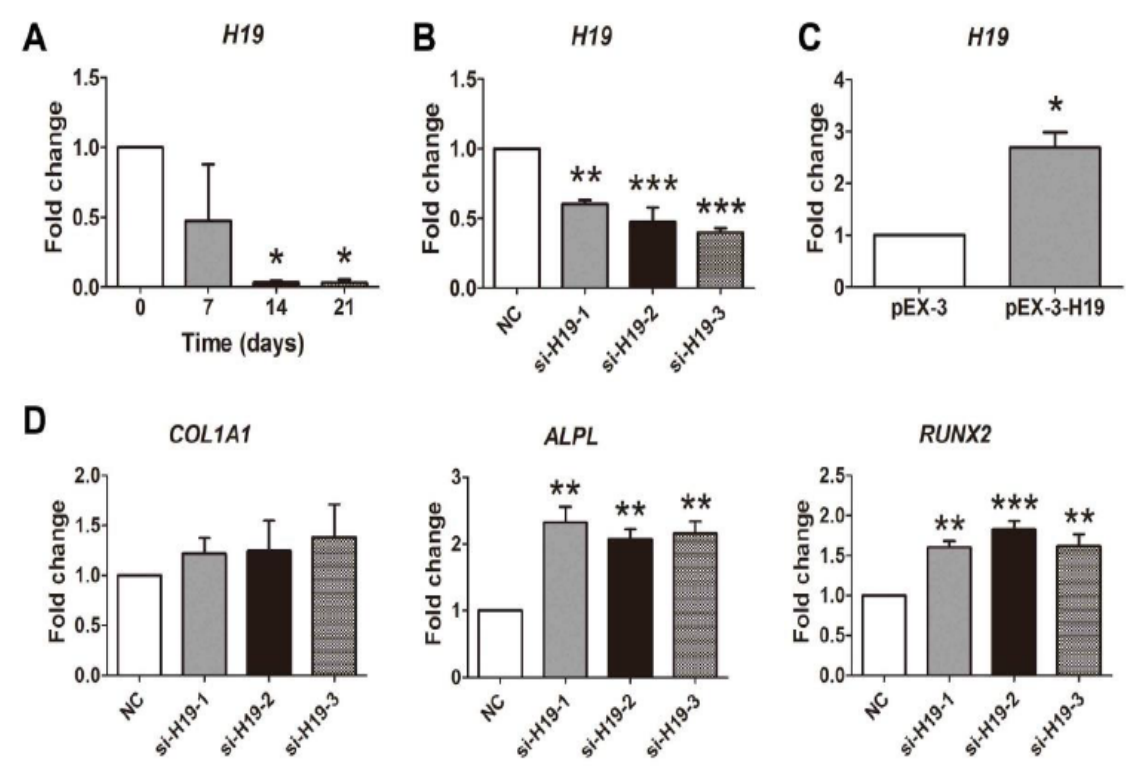

$E$

COL1A1

ALPL

RUNX2
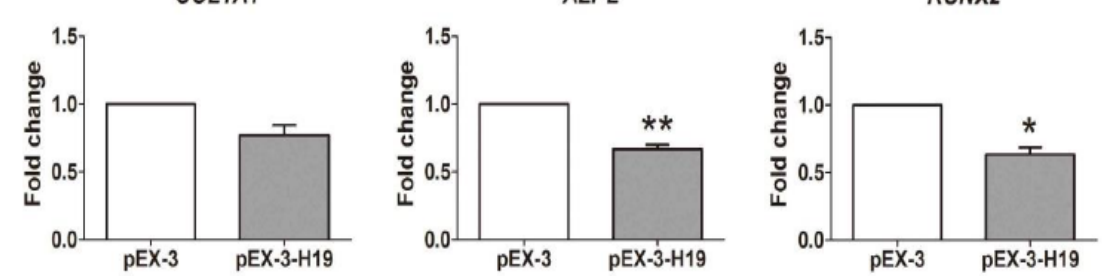

Fig. 6. Expression and function of $\mathrm{H} 19$ during osteogenic differentiation of human ASCs. A. Human ASCs were induced towards osteoblast differentiation for 7, 14 and 21 days, and expression of H19 was determined by qRT-PCR and compared to that at day 0. B-E. Human ASCs were transfected with scramble siRNA (NC) or H19 siRNA (si-H19-1, si-H19-2 and si-H19-3) or pEX-3 empty plasmid (pEX-3) or pEX-3-H19 for 48 hours, followed by induction of osteogenic differentiation for 3 days. Gene expression of H19 (B and C), and COL1A1, ALPL and RUNX2 (D and E) were measured by qRT-PCR and compared to that of NC or pEX3. Gene expression was normalized to GAPDH expression and calculated by the $2-\Delta \Delta \mathrm{Ct}$ method. Data was presented as mean \pm SD of three independent experiments. ${ }^{*} \mathrm{P}<0.05,{ }^{*} \mathrm{P}<0.01,{ }^{*} * \mathrm{P}<0.001$ represent significant differences relative to control groups as determined by one-way ANOVA followed by Dunnett- $t$ test or paired t-test.

\section{qRT-PCR validation}

In order to validate our microarray results, the expression of five upregulated IncRNAs (SAA3P, LOC284080, XLOC_001453, XLOC_011980 and HHIP-AS1) and five downregulated IncRNAs (XLOC_007489, CTC-564N23.3, XLOC_010397, XLOC_002619 and LINC00638) with fold changes $>10.0$ in the microarray profile were examined by qRT-PCR in four osteogenic differentiated human ASC cultures and paired undifferentiated cultures. Although the qRT-PCR results failed to confirm the expression pattern of XLOC_001453 and XLOC_002619, results for the other eight lncRNAs exhibited similar trends as those observed in the microarray data. Furthermore, the changes determined by qRT-PCR were significant $(P<0.05)$ and the fold changes measured by qRT-PCR correlated with that from microarray $(r=0.6121, P=0.0334)$, demonstrating consistency between the qRT-PCR and microarray results (Fig. 4). 
Table 6. List of core regulatory lncRNAs in CNC network

\begin{tabular}{|c|c|c|c|c|c|c|c|}
\hline Seq name & $\begin{array}{l}\text { Chrom- } \\
\text { osome }\end{array}$ & Str-and & Start & End & Fold change & Regul-ation & $P$-value \\
\hline TCONS_00016098 & $\operatorname{chr} 9$ & + & 102648727 & 102657226 & -53.13 & down & 0.00000011 \\
\hline TCONS_00021794 & $\operatorname{chr} 13$ & + & 53631290 & 53650848 & -15.67 & down & 0.0000026 \\
\hline ENST00000512213 & $\operatorname{chr} 5$ & + & 76382625 & 76401633 & -15.11 & down & 0.00000090 \\
\hline TCONS_00022189 & $\operatorname{chr} 13$ & + & 64201007 & 64209643 & -11.50 & down & 0.0000031 \\
\hline ENST000000533267 & $\operatorname{chr} 11$ & - & 18134172 & 18137625 & +36.57 & up & 0.000000052 \\
\hline ENST00000531617 & $\operatorname{chr} 17$ & - & 72208812 & 72209447 & -54.85 & down & 0.0000062 \\
\hline ENST00000563280 & $\operatorname{chr} 16$ & - & 86598750 & 86601367 & +26.52 & up & 0.0000026 \\
\hline NR_024396 & $\operatorname{chr} 14$ & + & 105287537 & 105290055 & -15.24 & down & 0.0000059 \\
\hline ENST00000581971 & $\operatorname{chr} 18$ & - & 73556879 & 73580207 & +12.40 & up & 0.000018 \\
\hline TCONS_00029072 & chr21 & - & 9826937 & 9827584 & +53.65 & up & 0.00000087 \\
\hline TCONS_00025773 & chr17 & - & 76613497 & 76614757 & -12.70 & down & 0.00000082 \\
\hline ENST'00000561386 & $\operatorname{chr} 15$ & + & 62022048 & 62023588 & +23.36 & up & 0.0000055 \\
\hline
\end{tabular}

Construction of CNC network

In order to explore interactions among differentially expressed lncRNAs and mRNAs, a CNC network was constructed based on 55 upregulated and 33 downregulated lncRNAs exhibiting $>10$-fold changes in all four pairs of samples. Pearson's correlation for each differentially expressed lncRNA and its paired mRNA was calculated and those transcripts with coefficients $>0.999$ were chosen to build the network. In the CNC network, one lncRNA was able to correlate with different mRNAs, and vice versa. Transcripts exhibiting correlations with larger numbers of genes displayed greater degrees of connectivity and were considered as core regulatory factors. The lncRNAs with degrees $\geq 40$ were further selected to construct a more visible network. The final CNC network comprised 12 lncRNAs and 157 mRNAs, with 645 network pairs (Fig. 5). Both positive and negative correlations between lncRNAs and mRNAs were present, with detailed information regarding core regulatory lncRNAs shown in Table 6.

\section{Effects of IncRNA H19 on human ASCs osteogenesis}

The expression of $H 19$, which is upregulated and exhibits functions involved in osteogenic differentiation of human BMSCs $[16,18,20]$, was decreased (Fold change: 3.81 ) in osteogenic differentiated ASCs as compared with undifferentiated cells according to our microarray results. To validate the differences in these results and explore the possible function of $H 19$ in ASCs, we measured dynamic H19 expression during osteogenic processes of human ASCs and examined the effect of $H 19$ knockdown on osteogenesis of human ASCs. Consistent with our microarray results, expression of $H 19$ was decreased significantly at days 7, 14 and 21 when compared with day 0 (Fig. 6A). Additionally, qRT-PCR results confirmed significant inhibition of $H 19$ expression following siRNA transfection, and significant enhancement of H19 expression following transfection with pEX-3-H19 plasmid (Fig. 6B, C). Furthermore, knockdown of $H 19$ resulted in significantly increased expression of RUNX2 and ALPL, but not COL1A1, during osteogenic induction of human ASCs (Fig. 6D), whereas a significant decrease in expression of RUNX2 and ALPL was observed after H19 overexpression (Fig. 6E).

\section{Discussion}

An understanding of the molecular mechanisms associated with osteogenesis is fundamental to maintaining osteogenic capacity and realizing the therapeutic potential of human ASCs. Although the functional roles of transcriptional factors, microRNAs and epigenetic factors in osteogenesis have been extensively studied [30-34], few studies focused on the role and the underlying mechanisms of lncRNAs in this biological process [35]. Moreover, there is no report regarding genome-wide expression and function of IncRNAs in osteogenesis of ASCs. In the current study, we revealed the global expression profile of lncRNAs during osteogenic differentiation of human ASCs by microarray and predicted the potential function of the differentially expressed lncRNAs using bioinformatics analysis. In addition, our experimental evidence indicated that lncRNAs may play an important role in osteogenesis of human ASCs. 
We selected 10 lncRNAs for qRT-PCR validation of the microarray data. It was noticed that there was variations between IncRNA expression detected by qRT-PCR versus microarray. One explanation may be the inherent pitfalls in the differences between the two methods, including the different normalization procedures and priming methods used for reverse transcription [36]. However, expression of most of the lncRNAs selected for qRTPCR validation showed similar trends as those observed in the microarray results. Moreover, statistical analysis indicated that the differences in lncRNA expression were significant between differentiated and undifferentiated human ASCs and the fold changes measured by qRT-PCR significantly correlated with those from microarray. Therefore, the qRT-PCR results confirmed the reliability of the microarray data.

Expression of some lncRNAs is altered during osteogenic differentiation of other kinds of MSCs [18, 21,22]. Wang et al. [18] showed that 687 lncRNAs were significantly upregulated and 519 lncRNAs were significantly downregulated during osteogenesis of human BMSCs. Compared with those findings, we observed much more lncRNAs differentially expressed during osteogenesis of human ASCs. This might be explained by the different cell types used between the two studies, given that IncRNA expression is cell specific [14]. Another explanation could be the differences in microarray platforms utilized in the two studies. That used in this study included 30,586 lncRNAs and detected 17,246 lncRNAs expressed in human ASCs. Moreover, some previous studies investigated the function of particular lncRNA in osteogenesis. It was reported that suppression of IncRNA-ANCR could promote osteogenic differentiation of BMSCs [17]. However, we observed that expression of IncRNAANCR decreased slightly and without significance (Fold change: $1.14, P=0.080$ ) during osteogenesis of human ASCs according to our microarray results. Whether lncRNA-ANCR also plays an inhibitory role during osteogenic differentiation of ASCs requires further research. Recently, the Zhou group [23] reported that IncRNA-MIR31HG was induced by nuclear factor (NF)- $\kappa B$ activation in human ASCs and that lncRNA-MIR31HG, in turn, contributed to NF- $\kappa B$ activation, which further inhibited bone formation under inflammatory conditions. More importantly, in our current study, we found that IncRNA-MIR31HG was significantly downregulated (Fold change: 2.31, $P=0.000066$ ) in osteogenic differentiated human ASCs when compared with undifferentiated cells. Therefore, these results suggested that during normal osteogenic differentiation of ASCs, downregulation of IncRNA-MIR31HG may be required, whereas in an inflammatory environment, MIR31HG may be pathologically upregulated to suppress bone formation. However, more specific experiments are required to test this hypothesis.

$H 19$, one of the most well-known imprinted genes, encodes a $2.3-\mathrm{kb}$ polyadenylated RNA that does not produce any known protein [37]. Several studies reported that $H 19$ was upregulated during osteogenesis in BMSCs $[16,18,20]$. In addition, $H 19$ promotes osteogenic differentiation of BMSCs via the transforming growth factor $\beta-1 / \mathrm{Smad} 3 /$ histone deacetylase pathway, with microRNA (miR) -675 derived from $H 19$ partially responsible for this pro-osteogenic effect [16]. However, another study showed that instead of promoting osteogenesis, miR-675-5p negatively regulates osteogenesis of BMSCs. The authors argued that this might be due to the negative-feedback loop associated with $H 19$ and miR-675-5p. Meanwhile, they revealed another mechanism for $H 19$ in the enhancement of osteogenic differentiation of BMSCs that concerned H19-mediated activation of Wnt signaling by acting as a microRNA sponge for miR-141 and miR-22, both of which negatively regulated $\beta$-catenin [20]. Here, we found that $H 19$ expression decreased significantly during osteogenic differentiation of human ASCs. To the best of our knowledge, only one study showed reduced expression of $H 19$ in ASCs as compared with BMSCs [38]; however, no report exists regarding $H 19$ expression during osteogenesis of ASCs. This discrepancy might be explained by evidence showing that $H 19$ expression is regulated in a tissue- and cell-specific manner during embryogenesis [39]. However, the specific role of H19 in ASC osteogenesis has not been studied and it might be different from that of BMSCs, given its different expression patterns observed between the two cell types. In the present study, we showed that at early stage of osteogenesis of human ASCs, knockdown of $\mathrm{H} 19$ led to upregulation of osteogenesis- 
related genes, including RUNX2 and $A L P L$, while overexpression of $H 19$ played a contrasting role. These findings suggested a negative regulatory role for $H 19$ in early osteogenesis of human ASCs. It was reported that $H 19$ also served as a competing endogenous RNA for the let-7 family of microRNAs and its deletion resulted in overexpression of let-7 [40]. Moreover, let-7 has been showed to enhance osteogenesis and suppress adipogenesis by negatively regulating high mobility group AT hook 2 (HMGA2) in ASCs [41]. Therefore, we attempted to determine whether $H 19$ could act as a molecular sponge for let-7 to regulate the function of HMGA2 in ASCs. However, manipulation of $\mathrm{H} 19$ in vitro has no significant effects on expression of let-7 or HMGA2 during early stage of osteogenic differentiation of human ASCs (data not shown), which may imply that let-7 and HMGA2 are not targets of H19 during ASC osteogenesis. Therefore, further investigation to determine the direct targets and detailed mechanism of $H 19$ during osteogenesis of human ASCs is required.

There were some limitations in the present study. First, we only chose cells after a 14-day osteogenic induction as osteogenic differentiated cells. This might result in losing information about the dynamic pattern of IncRNA expression over the course of the entire osteogenic process. However, this level of testing would be costly and increase the difficulty in comparing the gene expression profiles of different time points using microarray analysis. On the other hand, comparison of differentiated and undifferentiated stem cells has been widely used to investigate the differentiation process. Moreover, we monitored the expression of $H 19$ at different time points during osteogenic induction and will measure the dynamic expression patterns of other candidate IncRNAs in future studies. Another limitation was that we did not identify the direct target of H19. Nonetheless, we have provided evidence of the potential role of $\mathrm{H19}$ in early osteogenesis of human ASCs. Further studies will be conducted to investigate the detailed mechanism. Finally, although the study identifies $\mathrm{H} 19$ as a negative regulator of human ASC osteogenic differentiation, further studies are still required to determine what impact it has on matrix mineralization by ASC-derived osteoblasts.

In summary, to our knowledge, this is the first study revealing the comprehensive expression profiles of IncRNAs during osteogenic differentiation of human ASCs. The potential functions and mechanisms of the dysregulated lncRNAs were also investigated by bioinformatics analysis and lncRNA transfection experiments. Although the specific role and mechanism of these candidate IncRNAs in osteogenesis of human ASCs require further studies, our findings extended the information known about the osteogenic process of ASCs and suggested directions for further investigations on the regulation of osteoblastic differentiation of ASCs as well as treatments for osteoporosis and other bone diseases.

\section{Acknowledgements}

We thank Dr. Aishan He, Zibo Yang, Baoxi Yu and Guping Mao at Sun Yat-sen University (Guangzhou, China) for valuable assistance.

This study was supported by National Natural Science Foundation of China (81672198, 81472101, 81672145, 81371941 and 81572119), Science and Technology Planning Project of Guangdong Province, China (2013B021800119, 2015A020212015 and 2016A020214005), and the Basic Scientific Training Project for Young Medical Teachers of Sun Yat-sen University (15ykpy11). The sponsors were not involved in the design of the study; collection, analysis and interpretation of data or writing of the manuscript.

\section{Disclosure Statement}

The authors declare that they have no competing interests. 


\section{Cellular Physiology Cell Physiol Biochem 2017;42:1037-1050 \begin{tabular}{c|l} 
and Biochemistry Published online: June 28, 2017 & $\begin{array}{l}\text { C } 2017 \text { The Author(s). Published by S. Karger AG, Basel } \\
\text { www.karger.com/cpb }\end{array}$ \\
\hline
\end{tabular}}

Huang et al.: LncRNAs in Osteogenesis of Human ASCs

\section{References}

1 Lane NE: Epidemiology, etiology, and diagnosis of osteoporosis. Am J Obstet Gynecol 2006;194:S3-11.

-2 Ito H: Clinical considerations of regenerative medicine in osteoporosis. Curr Osteoporos Rep 2014;12:230234.

3 Antebi B, Pelled G, Gazit D: Stem cell therapy for osteoporosis. Curr Osteoporos Rep 2014;12:41-47.

-4 You L, Pan L, Chen L, Gu W, Chen J: MiR-27a is Essential for the Shift from Osteogenic Differentiation to Adipogenic Differentiation of Mesenchymal Stem Cells in Postmenopausal Osteoporosis. Cell Physiol Biochem 2016;39:253-265.

5 Mueller SM, Glowacki J: Age-related decline in the osteogenic potential of human bone marrow cells cultured in three-dimensional collagen sponges. J Cell Biochem 2001;82:583-590.

6 Stenderup K, Justesen J, Clausen C, Kassem M: Aging is associated with decreased maximal life span and accelerated senescence of bone marrow stromal cells. Bone 2003;33:919-926.

7 Rodriguez JP, Montecinos L, Rios S, Reyes P, Martinez J: Mesenchymal stem cells from osteoporotic patients produce a type I collagen-deficient extracellular matrix favoring adipogenic differentiation. J Cell Biochem 2000;79:557-565.

8 Tapp H, Hanley EN, Jr., Patt JC, Gruber HE: Adipose-derived stem cells: characterization and current application in orthopaedic tissue repair. Exp Biol Med (Maywood) 2009;234:1-9.

-9 Chen HT, Lee MJ, Chen CH, Chuang SC, Chang LF, Ho ML, Hung SH, Fu YC, Wang YH, Wang HI, Wang GJ, Kang L, Chang JK: Proliferation and differentiation potential of human adipose-derived mesenchymal stem cells isolated from elderly patients with osteoporotic fractures. J Cell Mol Med 2012;16:582-593.

10 Zhu M, Kohan E, Bradley J, Hedrick M, Benhaim P, Zuk P: The effect of age on osteogenic, adipogenic and proliferative potential of female adipose-derived stem cells. J Tissue Eng Regen Med 2009;3:290-301.

11 Mirsaidi A, Genelin K, Vetsch JR, Stanger S, Theiss F, Lindtner RA, von Rechenberg B, Blauth M, Muller R, Kuhn GA, Hofmann Boss S, Ebner HL, Richards PJ: Therapeutic potential of adipose-derived stromal cells in age-related osteoporosis. Biomaterials 2014;35:7326-7335.

12 Guttman M, Amit I, Garber M, French C, Lin MF, Feldser D, Huarte M, Zuk O, Carey BW, Cassady JP, Cabili MN, Jaenisch R, Mikkelsen TS, Jacks T, Hacohen N, Bernstein BE, Kellis M, Regev A, Rinn JL, Lander ES: Chromatin signature reveals over a thousand highly conserved large non-coding RNAs in mammals. Nature 2009;458:223-227.

13 Ponting CP, Oliver PL, Reik W: Evolution and functions of long noncoding RNAs. Cell 2009;136:629-641.

14 Li YP, Wang Y: Large noncoding RNAs are promising regulators in embryonic stem cells. J Genet Genomics 2015;42:99-105.

15 Cui Y, Lu S, Tan H, Li J, Zhu M, Xu Y: Silencing of Long Non-Coding RNA NONHSAT009968 Ameliorates the Staphylococcal Protein A-Inhibited Osteogenic Differentiation in Human Bone Mesenchymal Stem Cells. Cell Physiol Biochem 2016;39:1347-1359.

16 Huang Y, Zheng Y, Jia L, Li W: Long Noncoding RNA H19 Promotes Osteoblast Differentiation Via TGFbeta1/Smad3/HDAC Signaling Pathway by Deriving miR-675. Stem Cells DOI:10.1002/stem.2225.

17 Zhu L, Xu PC: Downregulated LncRNA-ANCR promotes osteoblast differentiation by targeting EZH2 and regulating Runx2 expression. Biochem Biophys Res Commun 2013;432:612-617.

18 Wang L, Wang Y, Li Z, Li Z, Yu B: Differential expression of long noncoding ribonucleic acids during osteogenic differentiation of human bone marrow mesenchymal stem cells. Int Orthop 2015;39:10131019.

19 Zhuang W, Ge X, Yang S, Huang M, Zhuang W, Chen P, Zhang X, Fu J, Qu J, Li B: Upregulation of lncRNA MEG3 Promotes Osteogenic Differentiation of Mesenchymal Stem Cells From Multiple Myeloma Patients By Targeting BMP4 Transcription. Stem Cells 2015;33:1985-1997.

20 Liang WC, Fu WM, Wang YB, Sun YX, Xu LL, Wong CW, Chan KM, Li G, Waye MM, Zhang JF: H19 activates Wnt signaling and promotes osteoblast differentiation by functioning as a competing endogenous RNA. Sci Rep 2016;6:20121.

21 Zuo C, Wang Z, Lu H, Dai Z, Liu X, Cui L: Expression profiling of IncRNAs in C3H10T1/2 mesenchymal stem cells undergoing early osteoblast differentiation. Mol Med Rep 2013;8:463-467.

-22 Dong R, Du J, Wang L, Wang J, Ding G, Wang S, Fan Z: Comparison of long noncoding RNA and mRNA expression profiles in mesenchymal stem cells derived from human periodontal ligament and bone marrow. Biomed Res Int 2014;2014:317853. 


\section{Cellular Physiology Cell Physiol Biochem 2017;42:1037-1050

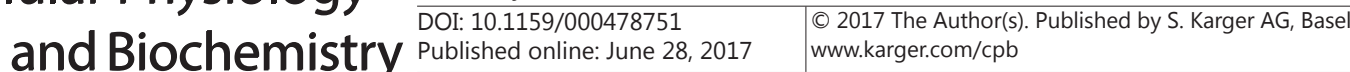

Huang et al.: LncRNAs in Osteogenesis of Human ASCs

-23 Jin C, Jia L, Huang Y, Zheng Y, Du N, Liu Y, Zhou Y: Inhibition of IncRNA MIR31HG Promotes Osteogenic Differentiation of Human Adipose-Derived Stem Cells. Stem Cells DOI:10.1002/stem.2439.

-24 Gamez B, Rodriguez-Carballo E, Graupera M, Rosa JL, Ventura F: Class I PI-3-Kinase Signaling Is Critical for Bone Formation Through Regulation of SMAD1 Activity in Osteoblasts. J Bone Miner Res 2016;31:16171630.

-25 Kim JH, Yoon SM, Song SU, Park SG, Kim WS, Park IG, Lee J, Sung JH: Hypoxia Suppresses Spontaneous Mineralization and Osteogenic Differentiation of Mesenchymal Stem Cells via IGFBP3 Up-Regulation. Int J Mol Sci 2016;17:

-26 Xi JC, Zang HY, Guo LX, Xue HB, Liu XD, Bai YB, Ma YZ: The PI3K/AKT cell signaling pathway is involved in regulation of osteoporosis. J Recept Signal Transduct Res 2015;35:640-645.

-27 Faghihi MA, Wahlestedt C: Regulatory roles of natural antisense transcripts. Nat Rev Mol Cell Biol 2009;10:637-643.

-28 Orom UA, Derrien T, Beringer M, Gumireddy K, Gardini A, Bussotti G, Lai F, Zytnicki M, Notredame C, Huang Q Guigo R, Shiekhattar R: Long noncoding RNAs with enhancer-like function in human cells. Cell 2010;143:46-58.

29 Cabili MN, Trapnell C, Goff L, Koziol M, Tazon-Vega B, Regev A, Rinn JL: Integrative annotation of human large intergenic noncoding RNAs reveals global properties and specific subclasses. Genes Dev 2011;25:1915-1927.

-30 Yang L, Ge D, Cao X, Ge Y, Chen H, Wang W, Zhang H: MiR-214 Attenuates Osteogenic Differentiation of Mesenchymal Stem Cells via Targeting FGFR1. Cell Physiol Biochem 2016;38:809-820.

-31 Huang J, Meng Y, Liu Y, Chen Y, Yang H, Chen D, Shi J, Guo Y: MicroRNA-320a Regulates the Osteogenic Differentiation of Human Bone Marrow-Derived Mesenchymal Stem Cells by Targeting HOXA10. Cell Physiol Biochem 2016;38:40-48.

-32 Zhang H, Li L, Dong Q Wang Y, Feng Q Ou X, Zhou P, He T, Luo J: Activation of PKA/CREB Signaling is Involved in BMP9-Induced Osteogenic Differentiation of Mesenchymal Stem Cells. Cell Physiol Biochem 2015;37:548-562.

-33 Xiao WL, Zhang DZ, Fan CH, Yu BJ: Intermittent Stretching and Osteogenic Differentiation of Bone Marrow Derived Mesenchymal Stem Cells via the p38MAPK-Osterix Signaling Pathway. Cell Physiol Biochem 2015;36:1015-1025.

-34 Zhou N, Hu N, Liao JY, Lin LB, Zhao C, Si WK, Yang Z, Yi SX, Fan TX, Bao W, Liang X, Wei X, Chen H, Chen C, Chen Q Lin X, Huang W: HIF-1alpha as a Regulator of BMP2-Induced Chondrogenic Differentiation, Osteogenic Differentiation, and Endochondral Ossification in Stem Cells. Cell Physiol Biochem 2015;36:4460.

-35 Tye CE, Gordon JA, Martin-Buley LA, Stein JL, Lian JB, Stein GS: Could lncRNAs be the missing links in control of mesenchymal stem cell differentiation? J Cell Physiol 2015;230:526-534.

-36 Morey JS, Ryan JC, Van Dolah FM: Microarray validation: factors influencing correlation between oligonucleotide microarrays and real-time PCR. Biol Proced Online 2006;8:175-193.

-37 Brannan CI, Dees EC, Ingram RS, Tilghman SM: The product of the H19 gene may function as an RNA. Mol Cell Biol 1990;10:28-36.

-38 Ravid O, Shoshani O, Sela M, Weinstock A, Sadan TW, Gur E, Zipori D, Shani N: Relative genomic stability of adipose tissue derived mesenchymal stem cells: analysis of ploidy, H19 long non-coding RNA and p53 activity. Stem Cell Res Ther 2014;5:139.

39 Gabory A, Jammes H, Dandolo L: The H19 locus: role of an imprinted non-coding RNA in growth and development. Bioessays 2010;32:473-480.

40 Kallen AN, Zhou XB, Xu J, Qiao C, Ma J, Yan L, Lu L, Liu C, Yi JS, Zhang H, Min W, Bennett AM, Gregory RI, Ding Y, Huang Y: The imprinted H19 lncRNA antagonizes let-7 microRNAs. Mol Cell 2013;52:101-112.

41 Wei J, Li H, Wang S, Li T, Fan J, Liang X, Li J, Han Q, Zhu L, Fan L, Zhao RC: let-7 enhances osteogenesis and bone formation while repressing adipogenesis of human stromal/mesenchymal stem cells by regulating HMGA2. Stem Cells Dev 2014;23:1452-1463. 